\title{
Internal symmetry in the constitutive model of perfect elastoplasticity
}

\author{
Hong-Ki Hong*, Chein-Shan Liu \\ Department of Civil Engineering, National Taiwan University, Taipei 106-17, Taiwan
}

Received 8 February 1999; accepted 29 March 1999

\begin{abstract}
Internal symmetry in the constitutive model of perfect elastoplasticity is investigated here. Using homogeneous coordinates, we convert the non-linear model to a linear system $\dot{\mathbf{X}}=\mathbf{A X}$. In this way the inherent symmetry in the constitutive model of perfect elastoplasticity (in the on phase) is brought out. The underlying structure is found to be the cone of Minkowski spacetime $\mathbb{M}^{n+1}$ on which the proper orthochronous Lorentz group $\mathrm{SO}_{0}(n, 1)$ left acts. When the plasticity mechanism is shut off by the input path, the internal symmetry is switched to a translation group $T(n)$ acting on the closed disc $\mathbb{D}^{n}$ of Euclidean space $\mathbb{E}^{n}$. Based on the group properties a Cayley transformation is developed, which updates the stress points to be automatically on the yield surface at every time increment. These results (and their generalizations to more sophisticated models) are essential for computational plasticity. As an example, the results calculated using the group-preserving scheme and the exact constitutive solutions for a rectilinear path are compared. (C) 1999 Elsevier Science Ltd. All rights reserved.
\end{abstract}

Keywords: Perfect elastoplasticity; Internal symmetry; Minkowski spacetime; Lorentz group; Group-preserving scheme

\section{Introduction}

The study of plastic behavior of solid materials and structural members under complicated mechanical environment is a very important topic for engineering science and industrial practice. In the study a substantial role has been played by the constitutive relations of elastoplasticity, to which many theoretical and experimental contributions have been made. However, it seems to authors' knowledge that there was no attempt in open literature until recently to study symmetry groups in the constitutive models of plasticity.

A constitutive model of plasticity is said to possess internal symmetry if it retains the forms of expressions for certain plastic phenomena even after changes in the states of the model have occurred. The changes or transformations occurring in the states of the model which leave the forms unchanged may constitute a Lie

\footnotetext{
* Corresponding author. Tel.: + 886-2-2366-1931; fax: + 886-2-2362-2975.

E-mail address: hkhong@ce.ntu.edu.tw (H.-K. Hong)
} 


\begin{tabular}{|ll|}
\hline Nomenclature & \\
$\mathbf{q}, \mathbf{q}^{\mathrm{e}}, \mathbf{q}^{\mathrm{p}}$ & generalized strain, generalized elastic strain and generalized plastic strain \\
$k_{\mathrm{e}}, Q^{0}$ & generalized elastic modulus and generalized yield stress \\
$\mathbf{Q}$ & generalized stress \\
$q_{0}$ & equivalent generalized plastic strain \\
$q_{\mathrm{y}}$ & generalized yield strain \\
$\mathbb{E}^{n}, \mathbb{D}^{n}, \mathbb{B}^{n}, \mathbb{S}^{n-1}$ & Euclidean $n$-space, closed $n$-disc, closed $n$-ball and $n$-sphere \\
$\mathbb{M}^{n+1}, \mathbf{g}$ & Minkowski spacetime and Minkowski metric \\
$\mathbf{X}, X^{0}, \mathbf{X}^{\mathrm{s}}$ & augmented stress, temporal coordinate and spatial coordinates \\
$T(n), \mathrm{SO} \mathrm{O}_{0}(n, 1)$ & translation group and proper orthochronous Lorentz group \\
$\mathrm{so}(n, 1)$ & Lie algebra of proper orthochronous Lorentz group \\
$\mathbf{A}$ & control tensor \\
$\mathbf{W}, \mathrm{SO}(3)$ & three-dimensional spin tensor and three-dimensional proper rotation group \\
$\mathrm{d} X, \mathrm{~d} Q$ & Minkowskian length of dX and Euclidean length of dQ \\
$I_{\mathrm{on}}$ & on interval of plasticity \\
$t_{0}, t_{i}, t_{1}, t$ & zero-value time, initial time, time and current time \\
$\kappa, \Delta t$ & a parameter and time increment \\
$\mathbf{G}, \mathrm{Cay}(\kappa \mathbf{A})$ & Lorentz transformation tensor and Cayley transformation \\
\end{tabular}

group of transformations and are naturally linked with the invariance of certain conserved quantities. A procedure for parameter estimation (and model identification) with effective utilization of the invariance properties along the experimental path will be more capable of capturing key features during plastic deformation. The passage from a flow plasticity model to a computational plasticity scheme inhibits a symmetry group analysis since the scheme does not usually have the same group properties as the flow model. A numerical algorithm which preserves symmetry in time marching will have long-term stability and much higher efficiency and accuracy.

In fact, the most important invariance of a model of plasticity is that the changing stress states remain on the (subsequent) yield surface during plastic deformation. That is the so-called consistency condition. Once one finds internal symmetry in a model of plasticity, he ensures among others the fulfillment of the consistency condition.

In this paper we propose to approach the symmetry issue and the computational application at the simplest three-dimensional model - perfect elastoplasticity. Generalizations to more sophisticated models will be hinted in due course (Section 11). We analyze the constitutive model of perfect elastoplasticity and attempt to achieve a deeper understanding of its underlying structure; more precisely speaking, we explore the structure of Minkowski spacetime $\mathbb{M}^{n+1}$ and the proper orthochronous Lorentz group $\operatorname{SO}_{0}(n, 1)$ inherent in the model in the on phase and also address the symmetry switching between the on (i.e. elastoplastic) and off (i.e. elastic) phases. To this end we need an appropriate setting to make the presentation clearer yet simpler, and, therefore, choose here to formulate the constitutive model directly in vector form. Let us consider the following constitutive model:

$$
\begin{aligned}
& \dot{\mathbf{q}}=\dot{\mathbf{q}}^{\mathrm{e}}+\dot{\mathbf{q}}^{\mathrm{p}}, \\
& \dot{\mathbf{Q}}=k_{\mathrm{e}} \dot{\mathbf{q}}^{\mathrm{e}}, \\
& \mathbf{Q} \dot{q}_{0}=Q^{0} \dot{\mathbf{q}}^{\mathrm{p}},
\end{aligned}
$$




$$
\begin{aligned}
& \|\mathbf{Q}\| \leqslant Q^{0}, \\
& \dot{q}_{0} \geqslant 0, \\
& \|\mathbf{Q}\| \dot{q}_{0}=Q^{0} \dot{q}_{0} .
\end{aligned}
$$

Here $\mathbf{Q}$ and $\dot{\mathbf{q}}$ are a pair of dual vectors in $n$-dimensional Euclidean space $\mathbb{E}^{n} ; \mathbf{Q}=\operatorname{col}\left(Q^{1}, Q^{2}, \ldots, Q^{n}\right)=$ $\operatorname{col}\left(Q_{1}, Q_{2}, \ldots, Q_{n}\right)$ denotes the generalized stress vector and $\dot{\mathbf{q}}=\operatorname{col}\left(\dot{q}_{1}, \dot{q}_{2}, \ldots, \dot{q}_{n}\right)=\operatorname{col}\left(\dot{q}^{1}, \dot{q}^{2}, \ldots, \dot{q}^{n}\right)$ denotes the generalized strain rate vector. ${ }^{1}$ The above constitutive model is re-postulated from the celebrated Prandtl-Reuss equation formulated by Prandtl [3] and Reuss [4], which is well known as the simplest (yet rather useful) three-dimensional constitutive law for describing a class of linearly elastic-perfectly plastic materials. Further explanations of the notation and the model are delegated to the next section.

The vector space of $\mathbf{Q}$ is an ordinary Euclidean space, which is endowed with a positive definite inner product. Later on, in Section 4, an augmented vector space will be built and endowed with an indefinite inner product. Although plasticity models are known to be highly nonlinear, the perfect elastoplasticity model (1)-(6) will be analyzed in Section 3 and synthesized and converted in Section 5 into a two-phase linear system with an on-off switch, and will be further examined in Section 6 concerning its Minkowski spacetime structure. Owing to the implicit linearity, a Lorentz group of transformations for the augmented vectors is discovered in Section 7. A projective transformation for the generalized stress responses is then realized in Section 8. Sections 9 and 10 address computation, one for rectilinear paths and the other for general paths.

\section{Perfect elastoplasticity}

The generalized stress vector $\mathbf{Q}$ may coincide with the stress deviator at a material point (and its neighborhood in the external space) as in the Prandtl-Reuss case, ${ }^{2}$ or it may stand for the vector of stress resultants on a cross section of a structural member like a beam, column, beam-column connection, plate, shell, or machine part, etc., or it may be the restoring forces integrated at the nodes of finite elements of a discretized continuum. The generalized strain vector $\mathbf{q}$ and the generalized strain rate vector $\dot{\mathbf{q}}$ are assumed to be so small that no account is taken of the spinning effect and the rate effect as well as the inertia effect.

As usual, the norm of a vector, say $\mathbf{Q}$, in Euclidean space $\mathbb{E}^{n}$ is defined as $\|\mathbf{Q}\|:=\sqrt{\mathbf{Q}^{t} \mathbf{Q}}$ where $\mathbf{Q}^{\mathrm{t}} \mathbf{Q}$ denotes the Euclidean inner product $\sum_{i=1}^{n} Q_{i} Q^{i}$ and the superscript $\mathrm{t}$ represents the transpose. The generalized elastic modulus $k_{\mathrm{e}}>0$ and the generalized yield stress $Q^{0}>0$ are the only two characteristic constants of the model, which are determined experimentally. The boldfaced symbols $\mathbf{q}^{\mathbf{e}}$ and $\mathbf{q}^{\mathbf{p}}$ are the $n$-dimensional (Euclidean) (column) vectors of generalized elastic and plastic strains, respectively, whereas $q_{0}$ is a scalar called the equivalent generalized plastic strain, with $Q^{0} \dot{q}_{0}$ being the (specific) power of dissipation. All $\mathbf{q}, \mathbf{q}^{\mathrm{e}}, \mathbf{q}^{\mathrm{p}}, \mathbf{Q}$ and $q_{0}$ are functions of one and the same independent variable, which in most cases is taken either as the ordinary time or as the arc length of an input path; however, for convenience, the independent variable no matter what it is will be simply called (the external) time and given the symbol $t$. An overdot denotes differentiation with respect to time, that is $\mathrm{d} / \mathrm{d} t$. Without loss of generality it is also postulated that with the above differential

\footnotetext{
${ }^{1}$ The notation and terminology were used by Prager [1,2] and many other scholars.

${ }^{2}$ Model (1)-(6) describes only the deviatoric part of the Prandtl-Reuss behavior under small deformation. The volumetric part of the Prandtl-Reuss behavior is linearly elastic and is thus excluded from the present study in order to focus on the more interesting elastic-plastic behavior of the deviatoric part.
} 
model there is a time instant designated as $t=t_{0}$, called the zero-value (or annealed) time, before and at which the model is in the zero-value (or annealed) state in the sense that the relevant values $\mathbf{q}\left(t_{0}\right), \mathbf{q}^{\mathbf{e}}\left(t_{0}\right), \mathbf{q}^{\mathbf{p}}\left(t_{0}\right), \mathbf{Q}\left(t_{0}\right)$ and $q_{0}\left(t_{0}\right)$ are all zeros.

Eq. (1) decomposes the generalized strain rate into the elastic and plastic parts, and Eq. (2) states a linear relation for the elastic part whereas Eq. (3) is an associated plastic-flow rule for the plastic part. Inequality (4) specifies admissible generalized stresses; we often call $\|\mathbf{Q}\| \leqslant Q^{0}$ the admissible closed ball, $\|\mathbf{Q}\|>Q^{0}$ the forbidden exterior, and $\|\mathbf{Q}\|=Q^{0}$ the yield hypersphere. Inequality (5) or $Q^{0} \dot{q}_{0} \geqslant 0$ requires the (specific) power of dissipation be nonnegative. It is easy to comprehend and appreciate Eqs. (1)-(5); however, Eq. (6) may need more explanation. With the aid of Eqs. (4) and (5), it simply requires $q_{0}$ be frozen if $\|\mathbf{Q}\|<Q^{0}$, so that $\dot{q}_{0}=0$ drastically reduces Eqs. (1)-(3) to $\dot{\mathbf{Q}}=k_{\mathrm{e}} \dot{\mathbf{q}}$, a linearly elastic relation. The significance of the complementary trios (4)-(6) cannot be overemphasized. It furnishes the model with an on-off switch for the mechanism of plasticity, and the conditions to switch on and to switch off the mechanism can thus be derived in a very precise way. Therefore, depending upon the input path, the model can be either in the ON (or elastoplastic) phase governed by Eqs. (1)-(3) or in the OFF (or elastic) phase governed by $\dot{\mathbf{Q}}=k_{\mathrm{e}} \dot{\mathbf{q}}$, as will be made clear in the next section.

\section{Switch for the mechanism of plasticity}

In this section we analyze flow model (1)-(6), deriving the on-off switching criteria for the mechanism of plasticity [5].

In the study of rate-independent plasticity we are always concerned with paths. By a path we mean a continuous curve whose velocity vectors are piecewise continuous. From Eqs. (3), (5) and (6) and $Q^{0}>0$, it is not difficult to prove that

$$
\dot{q}_{0}=\left\|\dot{\mathbf{q}}^{\mathrm{p}}\right\|
$$

that is, $q_{0}$ is the arc length of a path in the generalized plastic strain space.

Substituting Eqs. (2) and (3) into Eq. (1) gives

$$
\frac{1}{k_{\mathrm{e}}} \dot{\mathbf{Q}}+\frac{\dot{q}_{0}}{Q^{0}} \mathbf{Q}=\dot{\mathbf{q}}
$$

or

$$
\frac{\mathrm{d}}{\mathrm{d} t}\left(X^{0} \mathbf{Q}\right)=k_{\mathrm{e}} X^{0} \dot{\mathbf{q}}
$$

where the integrating factor

$$
X^{0}:=\exp \left(\frac{q_{0}}{q_{\mathrm{y}}}\right)
$$

is called the internal time, in which the generalized yield strain is defined as

$$
q_{\mathrm{y}}:=\frac{Q^{0}}{k_{\mathrm{e}}} .
$$

Taking the Euclidean inner product of Eq. (8) with $\mathbf{Q}$ gives

$$
\frac{1}{k_{\mathrm{e}}} \mathbf{Q}^{\mathrm{t}} \dot{\mathbf{Q}}+\frac{\dot{q}_{0}}{Q^{0}} \mathbf{Q}^{\mathrm{t}} \mathbf{Q}=\mathbf{Q}^{\mathrm{t}} \dot{\mathbf{q}}
$$


which, due to the constancy of $Q^{0}$, asserts

$$
\|\mathbf{Q}\|=Q^{0} \Rightarrow \mathbf{Q}^{\mathrm{t}} \dot{\mathbf{q}}=Q^{0} \dot{q}_{0},
$$

or in terms of $X^{0}$ via Eq. (10),

$$
\|\mathbf{Q}\|=Q^{0} \Rightarrow \frac{\dot{X}^{0}}{X^{0}}=\frac{\mathbf{Q}^{\mathrm{t}}}{Q^{0}} \frac{\dot{\mathbf{q}}}{q_{\mathrm{y}}} .
$$

Since $Q^{0}>0$, we have by Eq. (13)

$$
\|\mathbf{Q}\|=Q^{0} \Rightarrow\left\{\mathbf{Q}^{\mathbf{t}} \dot{\mathbf{q}}>0 \Leftrightarrow \dot{q}_{0}>0\right\} .
$$

Thus we have deduced the " $\Rightarrow$ " part of the following statement:

$$
\left\{\|\mathbf{Q}\|=Q^{0} \quad \text { and } \quad \mathbf{Q}^{\mathrm{t}} \dot{\mathbf{q}}>0\right\} \Leftrightarrow \dot{q}_{0}>0 .
$$

On the other hand, if $\dot{q}_{0}>0$, Eq. (6) ensures $\|\mathbf{Q}\|=Q^{0}$, which together with Eq. (15) proves the " $\Leftarrow$ " part of the above statement. In other words, the yield condition $\|\mathbf{Q}\|=Q^{0}$ and the straining condition $\mathbf{Q}^{\mathrm{t}} \dot{\mathbf{q}}>0$ are sufficient and necessary for plastic irreversibility $\dot{q}_{0}>0$. Considering Eq. (16) together with Eqs. (4), (5), (7) and (13), we obtain the following on-off switching criteria for the mechanism of plasticity:

$$
\dot{q}_{0}=\left\|\dot{\mathbf{q}}^{\mathbf{p}}\right\|= \begin{cases}\frac{1}{Q_{0}} \mathbf{Q}^{\mathrm{t}} \dot{\mathbf{q}}>0 & \text { if }\|\mathbf{Q}\|=Q^{0} \text { and } \mathbf{Q}^{\mathbf{t}} \dot{\mathbf{q}}>0, \\ 0 & \text { if }\|\mathbf{Q}\|<Q^{0} \text { or } \mathbf{Q}^{\mathrm{t}} \dot{\mathbf{q}} \leqslant 0 .\end{cases}
$$

Based on the criteria and the complementary trios (4)-(6), there are precisely two phases: the ON phase in which $\dot{q}_{0}>0$ and $\|\mathbf{Q}\|=Q^{0}$ and the OFF phase in which $\dot{q}_{0}=0$ and $\|\mathbf{Q}\| \leqslant Q^{0}$; see Fig. 1 . In the on phase the mechanism of plasticity is on and the model exhibits elastoplastic behavior, which is irreversible, while in the off phase the mechanism of plasticity is off and the model responds elastically and reversibly.

\section{Minkowski spacetime}

Let us define

$$
\mathbf{X}=\left[\begin{array}{c}
\mathbf{X}^{\mathrm{s}} \\
X^{0}
\end{array}\right]=\left[\begin{array}{c}
X^{1} \\
X^{2} \\
\vdots \\
X^{n} \\
X^{0}
\end{array}\right]:=\frac{\exp \left(q_{0} / q_{\mathrm{y}}\right)}{Q^{0}}\left[\begin{array}{c}
\mathbf{Q} \\
Q^{0}
\end{array}\right]=\frac{\exp \left(q_{0} / q_{\mathrm{y}}\right)}{Q^{0}}\left[\begin{array}{c}
Q^{1} \\
Q^{2} \\
\vdots \\
Q^{n} \\
Q^{0}
\end{array}\right]
$$

and call it the $(n+1)$-dimensional augmented stress vector. Note that the last component of Eq. (18) is consistent with Eq. (10) and that the components of the generalized stress vector $\mathbf{Q}$ and the components of the augmented stress vector $\mathbf{X}$ are indeed the non-homogeneous and homogeneous coordinates of the same stress state. Two augmented stress vectors which only differ by a non-zero scalar multiple represent the same generalized stress vector.

Now we recast model (1)-(6) postulated in the generalized stress space of $\mathbf{Q}$ into a model in the augmented stress space of X. Accordingly, Eqs. (9), (6), (4), and (5) become 


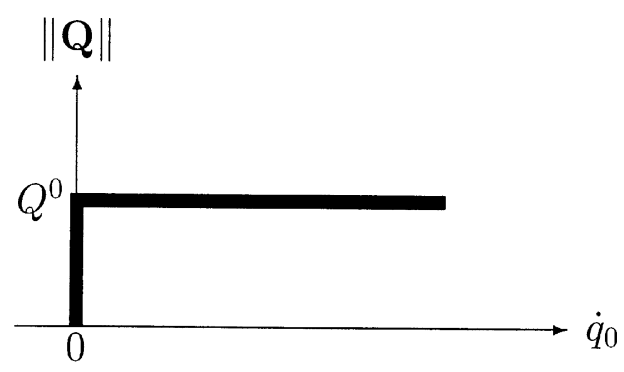

Fig. 1. The complementary trios (4)-(6) specify the admissible region in the phase plane of $\left(\dot{q}_{0},\|\mathbf{Q}\|\right)$, which is marked by heavy lines.

$$
\begin{aligned}
& {\left[\begin{array}{cc}
\mathbf{I}_{n} & \mathbf{0}_{n \times 1} \\
\mathbf{0}_{1 \times n} & \mathbf{X}^{\mathbf{t}} \mathbf{g X}
\end{array}\right] \dot{\mathbf{X}}=\frac{1}{q_{\mathrm{y}}}\left[\begin{array}{cc}
\mathbf{0}_{n \times n} & \dot{\mathbf{q}} \\
\mathbf{0}_{1 \times n} & 0
\end{array}\right] \mathbf{X},} \\
& \mathbf{X}^{\mathbf{t}} \mathbf{g X} \leqslant 0 \text {, } \\
& \dot{X}^{0} \geqslant 0 \text {, }
\end{aligned}
$$

in terms of the Minkowski metric (in the space-like convention)

$$
\mathbf{g}=\left[\begin{array}{ll}
\mathbf{g}_{\mathrm{ss}} & \mathbf{g}_{\mathrm{s} 0} \\
\mathbf{g}_{0 \mathrm{~s}} & \mathrm{~g}_{00}
\end{array}\right]=\left[\begin{array}{cc}
\mathbf{I}_{n} & \mathbf{0}_{n \times 1} \\
\mathbf{0}_{1 \times n} & -1
\end{array}\right],
$$

where $\mathbf{I}_{n}$ is the identity tensor of order $n$. The vector space of augmented stresses $\mathbf{X}$ endowed with the Minkowski metric tensor $\mathbf{g}$ is referred to as Minkowski spacetime and designated as $\mathbb{M}^{n+1}$.

Spacetime of this sort underlying the constitutive theory may be called internal spacetime, because we can think of it as having to do with the intrinsic nature of the mechanical behavior of the solid materials or the structural members which the constitutive model describes, rather than their position or motion in ordinary (external) space and (external) time. Thus the "temporal" coordinate $X^{0}$ and the "spatial" coordinates $\mathbf{X}^{\mathbf{s}}=\left(X^{1}, X^{2}, \ldots, X^{n}\right)$ may be thought of as the internal time and the internal space, respectively. In this treatment $X^{0}$ and $\mathbf{X}^{\mathrm{s}}$ are no more disparate and incompatible as the usual $(n+1)$-dimensional vector in Euclidean spacetime $\mathbb{E}^{n+1}$; they are now being organized to an integrated object in Minkowski spacetime $\mathbb{M}^{n+1}$, which is not a simple extension of ordinary Euclidean $n$-space to $n+1$ dimensions, with $X^{0}$ as just one more dimension. Because the corresponding entries in the metric have different signs, -1 versus positive definiteness, the "temporal" coordinate $X^{0}$ is not on the same footing as the $n$ "spatial" coordinates $\left(X^{1}, X^{2}, \ldots, X^{n}\right)$, and the structure built on the spacetime consequently has group properties (see Sections 7 and 8) quite unlike that on Euclidean space.

Regarding Eqs. (4) and (20), we may further distinguish two correspondences:

$$
\begin{aligned}
&\|\mathbf{Q}\|=Q^{0} \Leftrightarrow \mathbf{X}^{\dagger} \mathbf{g X}=0, \\
&\|\mathbf{Q}\|<Q^{0} \Leftrightarrow \mathbf{X}^{\mathbf{t}} \mathbf{g X}<0 .
\end{aligned}
$$

That is, a generalized stress vector $\mathbf{Q}$ on (resp. within) the yield hypersphere $\|\mathbf{Q}\|=Q^{0}$ in the generalized stress space of $\left(Q^{1}, Q^{2}, \ldots, Q^{n}\right)$ corresponds to an augmented stress vector $\mathbf{X}$ on the cone ${ }^{3}\left\{\mathbf{X} \mid \mathbf{X}^{\mathbf{t}} \mathbf{g X}=0\right\}$ of Minkowski spacetime (resp. in the interior ${ }^{4}\left\{\mathbf{X} \mid \mathbf{X}^{\mathbf{t}} \mathbf{g X}<0\right\}$ of the cone). The exterior ${ }^{5}\left\{\mathbf{X} \mid \mathbf{X}^{\mathbf{t}} \mathbf{g X}>0\right\}$ of the

\footnotetext{
${ }^{3}$ Following [6] we refer the null cone emanating from $\mathbf{X}=\mathbf{0}$ as the cone.

${ }^{4}$ The time-like region of $\mathbf{X}=\mathbf{0}$.

${ }^{5}$ The space-like region of $\mathbf{X}=\mathbf{0}$.
} 

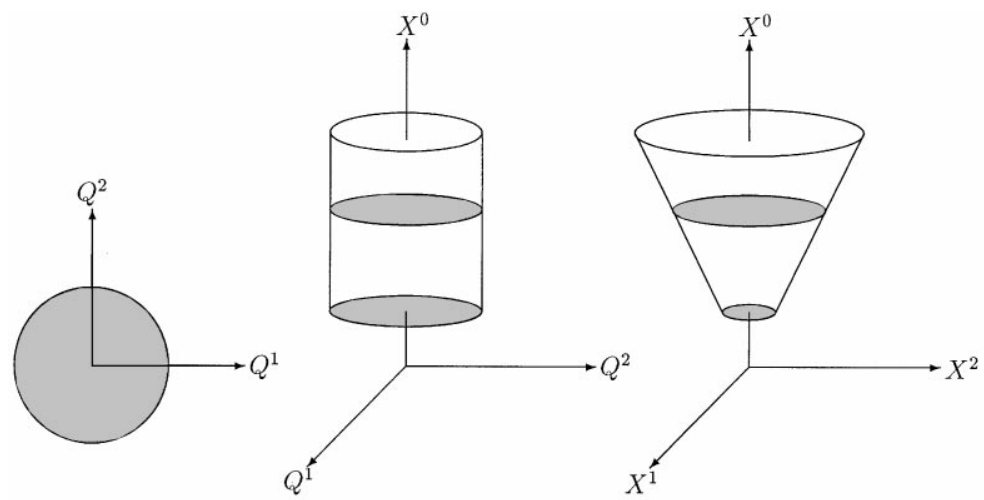

Fig. 2. The conventional concept of yield hypersphere $\mathbb{S}^{n-1}$ in the generalized stress space $\mathbf{Q}$ may be extended to a cylinder in the product space of $\left(\mathbf{Q}, X^{0}\right)$; however, it is the construction of the cone in Minkowski spacetime of $\mathbf{X}$ that signifies a conceptual breakthrough. A few discs of simultaneity are also shown.

cone is uninhabitable since $\|\mathbf{Q}\|>Q^{0}$ is forbidden according to axiom (4). Even though it admits an infinite number of Riemannian metrics, the yield hypersphere $\mathbb{S}^{n-1}$ in the $\mathbf{Q}$-space does not admit a Minkowskian metric, nor does the cylinder in the $\left(\mathbf{Q}^{t}, X^{0}\right)$-space. It is the cone in the $\mathbf{X}$-space which admits the Minkowski metric. As an illustration, a schematic plot is shown in Fig. 2.

Taking the Euclidean inner product of Eq. (9) with $\mathbf{Q}$ and substituting Eq. (18), we have

$$
\frac{1}{Q^{0} q_{\mathrm{y}}} \mathbf{Q}^{\mathrm{t}} \dot{\mathbf{q}}=\frac{1}{\left(X^{0}\right)^{2}} \frac{\mathrm{d}}{\mathrm{d} t}\left[\left(\mathbf{X}^{\mathrm{s}}\right)^{\mathrm{t}} \mathbf{g}_{\mathrm{ss}} \mathbf{X}^{\mathrm{s}}\right] \text {. }
$$

Upon considering $Q^{0}>0, q_{\mathrm{y}}>0$ and $X^{0} \geqslant 1$, we have

$$
\begin{gathered}
\mathbf{Q}^{\mathrm{t}} \dot{\mathbf{q}}>0 \Leftrightarrow \frac{\mathrm{d}}{\mathrm{d} t}\left[\left(\mathbf{X}^{\mathrm{s}}\right)^{\mathrm{t}} \mathbf{g}_{\mathrm{ss}} \mathbf{X}^{\mathrm{s}}\right]>0, \\
\mathbf{Q}^{\mathrm{t}} \dot{\mathbf{q}} \leqslant 0 \Leftrightarrow \frac{\mathrm{d}}{\mathrm{d} t}\left[\left(\mathbf{X}^{\mathrm{s}}\right)^{\mathrm{t}} \mathbf{g}_{\mathrm{ss}} \mathbf{X}^{\mathrm{s}}\right] \leqslant 0 .
\end{gathered}
$$

Hence in the augmented stress space what corresponds to the yield condition $\|\mathbf{Q}\|=Q^{0}$ is the cone condition $\mathbf{X}^{\mathbf{t}} \mathbf{g X}=0$ and what corresponds to the straining condition $\mathbf{Q}^{\mathbf{t}} \dot{\mathbf{q}}>0$ is the growing "spatial" radial coordinate condition d $\left[\left(\mathbf{X}^{\mathrm{s}}\right)^{t} \mathbf{g}_{\mathrm{ss}} \mathbf{X}^{\mathrm{s}}\right] / \mathrm{d} t>0$.

In view of Eqs. (18) and (23)-(26), the on-off switching criteria (17) become

$$
\begin{aligned}
& \dot{X}^{0}=\left(\mathbf{X}^{\mathrm{s}}\right)^{\mathrm{t}}\left(\dot{\mathbf{q}} / q_{\mathrm{y}}\right)>0 \text { if } \mathbf{X}^{\mathrm{t}} \mathbf{g X}=0 \text { and }(\mathrm{d} / \mathrm{d} t)\left[\left(\mathbf{X}^{\mathrm{s}}\right)^{\mathrm{t}} \mathbf{g}_{\mathrm{ss}} \mathbf{X}^{\mathrm{s}}\right]>0, \\
& \dot{X}^{0}=0 \text { if } \mathbf{X}^{\mathbf{t}} \mathbf{g X}<0 \text { or }(\mathrm{d} / \mathrm{d} t)\left[\left(\mathbf{X}^{\mathrm{s}}\right)^{\mathrm{t}} \mathbf{g}_{\mathrm{ss}} \mathbf{X}^{\mathrm{s}}\right] \leqslant 0,
\end{aligned}
$$

in the augmented stress space.

\section{Two-phase systems}

In this section, using the on-off switching criteria, we synthesize and convert flow model (1)-(6) to two-phase systems, searching for as revealing representations as possible. The following are three representations, respectively, in the $\mathbf{Q}$-spaces, the $\left(\mathbf{Q}, X^{0}\right)$-space, and the $\mathbf{X}$-space. The three spaces have been illustrated in Fig. 2. 


\subsection{Non-linear representation in the $\mathbf{Q}$-space}

Using Eq. (17) to eliminate $q_{0}$ from Eq. (8) results in a two-phase non-linear system of $n$ equations:

$$
\dot{\mathbf{Q}}= \begin{cases}-\left(\mathbf{Q}^{\mathrm{t}} / Q^{0}\right)\left(\dot{\mathbf{q}} / q_{\mathrm{y}}\right) \mathbf{Q}+k_{\mathrm{e}} \dot{\mathbf{q}} & \text { if }\|\mathbf{Q}\|=Q^{0} \text { and } \mathbf{Q}^{\mathrm{t}} \dot{\mathbf{q}}>0, \\ k_{\mathrm{e}} \dot{\mathbf{q}} & \text { if }\|\mathbf{Q}\|<Q^{0} \text { or } \mathbf{Q}^{\mathrm{t}} \dot{\mathbf{q}} \leqslant 0,\end{cases}
$$

of which the latter is linear and represents an instantaneous response, and the former is a system of nonlinear differential equations. This is a non-linear representation in the $n$-dimensional space of $\mathbf{Q}=\left(Q^{1}, Q^{2}, \ldots, Q^{n}\right)$

\subsection{Representation in the $\left(\mathbf{Q}, X^{0}\right)$-space}

In the $\left(\mathbf{Q}, q_{0}\right)$-space, or equivalently in the $\left(\mathbf{Q}, X^{0}\right)$-space, the two-phase system of $n+1$ Eqs. (8) and (17), is non-linear. However, we may arrange the solution process in two steps, making the problem "linear" in a certain sense.

The solution of Eq. (9) is

$$
\mathbf{Q}(t)=\frac{X^{0}\left(t_{i}\right)}{X^{0}(t)} \mathbf{Q}\left(t_{i}\right)+k_{\mathrm{e}} \int_{t_{i}}^{t} \frac{X^{0}(\xi)}{X^{0}(t)} \dot{\mathbf{q}}(\xi) \mathrm{d} \xi .
$$

Substituting this into Eq. (17), using Eqs. (10) and (11) to change $q_{0}$ to $X^{0}$, integrating, and changing the order of the double integral, we finally obtain [7]

$$
X^{0}(t)=\left\{\begin{array}{cl}
\left\{1+\left(1 / q_{\mathrm{y}} Q^{0}\right)\left[\mathbf{q}(t)-\mathbf{q}\left(t_{i}\right)\right]^{\mathrm{t}} \mathbf{Q}\left(t_{i}\right)\right\} X^{0}\left(t_{i}\right) & \\
+\left(1 / q_{\mathrm{y}}^{2}\right) \int_{t_{i}}^{t}[\mathbf{q}(t)-\mathbf{q}(\xi)]^{t} \dot{\mathbf{q}}(\xi) X^{0}(\xi) \mathrm{d} \xi & \text { if }\|\mathbf{Q}\|=Q^{0} \text { and } \mathbf{Q}^{\mathrm{t}} \dot{\mathbf{q}}>0 \\
X^{0}\left(t_{i}\right) & \text { if }\|\mathbf{Q}\|<Q^{0} \text { or } \mathbf{Q}^{\mathrm{t}} \dot{\mathbf{q}} \leqslant 0 .
\end{array}\right.
$$

The first of Eq. (30) is a linear Volterra integral equation. Thus, given the q-path, we first solve Eq. (30) for $X^{0}(t)$ and then calculate $\mathbf{Q}(t)$ via Eq. (29).

\subsection{Linear representation in the $\mathbf{X}$-space}

Organizing Eqs. (9), (27a) and (27b) with the aid of Eq. (18), we have the augmented stress (linear differential) equation

$$
\dot{\mathbf{X}}=\mathbf{A X}
$$

with the control tensor

$$
\begin{aligned}
& \mathbf{A}:=1 / q_{\mathrm{y}}\left[\begin{array}{cc}
\mathbf{0}_{n \times n} & \dot{\mathbf{q}} \\
\dot{\mathbf{q}}^{\mathrm{t}} & 0
\end{array}\right] \quad \text { if } \mathbf{X}^{\mathrm{t}} \mathbf{g X}=0 \text { and }(\mathrm{d} / \mathrm{d} t)\left[\left(\mathbf{X}^{\mathrm{s}}\right)^{\mathrm{t}} \mathbf{g}_{\mathrm{ss}} \mathbf{X}^{\mathrm{s}}\right]>0 \\
& \mathbf{A}:=1 / q_{\mathrm{y}}\left[\begin{array}{cc}
\mathbf{0}_{n \times n} & \dot{\mathbf{q}} \\
\mathbf{0}_{1 \times n} & 0
\end{array}\right] \quad \text { if } \mathbf{X}^{\mathrm{t}} \mathbf{g X}<0 \text { or }(\mathrm{d} / \mathrm{d} t)\left[\left(\mathbf{X}^{\mathrm{s}}\right)^{\mathrm{t}} \mathbf{g}_{\mathrm{ss}} \mathbf{X}^{\mathrm{s}}\right] \leqslant 0
\end{aligned}
$$

It is remarkable that in the augmented stress space the two-phase system becomes linear. All together it is a two-phase linear system with an on-off switch. The control tensor $\mathbf{A}$ in the on phase possesses the following properties: $\mathbf{A}^{\mathbf{t}}=\mathbf{A}, \operatorname{tr} \mathbf{A}=0, \operatorname{det} \mathbf{A}=0$, and its eigenvalues are 0 of multiplicity $n-1$ and $\pm\|\dot{\mathbf{q}}\| / q_{\mathrm{y}}$. As usual, 
the superscript $t$ stands for the transpose, the symbol tr for the trace, and det for the determinant. The last row of the off-phase's $\mathbf{A}$ is full of zeros since $X^{0}$ is constant (i.e., $q_{0}$ is fixed) in the off phase. Thus we have revealed the linearity of the perfect elastoplasticity model both in the on and off phases.

\subsection{Comments}

The multi-phase non-linear problems of plasticity were usually treated by workers in plasticity with various numerical schemes, which often encounter the tremendous difficulties of plastic non-linearity and yield inconsistency. The passage directly from flow model (1)-(6) to a numerical scheme, if no care is taken of, may alter or destroy the underlying structure of the model, resulting in unstable, inefficient, and inaccurate calculations. The passage from the above three representations to numerical schemes has the merit of the automatic fulfillment of the consistency condition. However, a numerical scheme based on the representation (28) in the $\mathbf{Q}$-space suffers from non-linearity. A numerical scheme based on the representation in the $\left(\mathbf{Q}, X^{0}\right)$-space, being non-linear but essentially "linear" in the above sense, has been shown to be much more efficient [8].

Comparing the two representations (28) and (31), we observe that Eq. (28) is non-linear and of the $n$ order, but Eq. (31) is linear and of the $n+1$ order; hence, the implicit linearity is unfolded at the expense of raising one order up. The representation in the $\mathbf{X}$-space is linear, as just said, and, moreover, easy to retain the internal symmetries of the model, thus facilitating the fulfillment of the consistency condition. Therefore, we will focus on the linear representation in the $\mathbf{X}$-space in the remainder of the paper.

\section{Properties of paths in Minkowski spacetime}

The augmentation of the states in $n$ dimensions to $n+1$ dimensions is far more than a mere mathematical artifice. In this section the rich properties of the paths admissible in Minkowski spacetime will be explored. Criteria (17) ensure that

$$
\dot{q}_{0} \mathbf{Q}^{\mathrm{t}} \mathbf{\mathbf { Q }}=0
$$

no matter whether in the on or in the off phase. In view of Eqs. (3), (7) and (5) and of the positivity of the two characteristic constants, Eqs. (8) and (33) become, respectively,

$$
\begin{aligned}
& \frac{1}{k_{\mathrm{e}}} \dot{\mathbf{Q}}+\dot{\mathbf{q}}^{\mathrm{p}}=\dot{\mathbf{q}}, \\
& \left(\dot{\mathbf{q}}^{\mathrm{p}}\right)^{\mathrm{t}} \dot{\mathbf{Q}}=0,
\end{aligned}
$$

which together indicate a right-angled triangle; therefore, according to the Pythagorean theorem,

$$
\begin{aligned}
& 0 \leqslant \dot{q}_{0}=\left\|\dot{\mathbf{q}}^{\mathrm{p}}\right\| \leqslant\|\dot{\mathbf{q}}\|, \\
& \mathrm{d} Q:=\|\mathrm{d} \mathbf{Q}\|=k_{\mathrm{e}} \sqrt{\|\mathrm{d} \mathbf{q}\|^{2}-\left(\mathrm{d} q_{0}\right)^{2}}, \\
& \left(\dot{\mathbf{q}}^{\mathrm{p}}\right)^{\mathrm{t}} \dot{\mathbf{q}} \geqslant 0,
\end{aligned}
$$

all of which are valid for both the on and off phases, even though the triangle may degenerate to a line segment or even to a single point. What do these important observations (36)-(38) imply for a path in the Minkowski spacetime of augmented stresses? 


\subsection{Time-like paths are not allowed}

We first examine Eq. (36), which tells us that the minimum and maximum values of the (specific) dissipation power $\dot{\Lambda}(t)=Q^{0} \dot{q}_{0}(t)$ an admissible path in the generalized stress space may discharge are zero and $Q^{0}\|\dot{\mathbf{q}}(t)\|$, respectively. From Eqs. (36), (5), (10) and (11), it follows that

$$
\left(X^{0}\right)^{2} \dot{\mathbf{q}}^{\mathrm{t}} \dot{\mathbf{q}}-q_{\mathrm{y}}^{2}\left(\dot{X}^{0}\right)^{2} \geqslant 0 .
$$

Substituting Eqs. (9), (18) and (22) into the above equation, we obtain

$$
\dot{\mathbf{X}}^{t} \mathbf{g} \dot{\mathbf{X}} \geqslant 0 \text {. }
$$

Thus

$$
(\mathrm{d} X)^{2}:=\mathrm{d} \mathbf{X}^{\mathrm{t}} \mathbf{g} \mathrm{d} \mathbf{X} \geqslant 0 .
$$

Be cautious not to mix up $\mathrm{d} X$ with $\mathrm{d} X^{0}$. The former is the Minkowskian length of the path increment $\mathrm{d} \mathbf{X}$, while the latter is the "temporal" component of $\mathrm{d} \mathbf{X}$. Recalling that a path such that $\mathrm{d} \mathbf{X}^{\mathrm{t}} \mathbf{g} \mathrm{d} \mathbf{X}>0$ (resp. $=0$, $<0$ ) is called a space-like (resp. null, time-like) path in $\mathbb{M}^{n+1}$, we thereby conclude that the curve $\mathbf{X}\left(t^{\prime}\right)$, $t_{i}<t^{\prime} \leqslant t$, in the augmented stress space is a space-like or null path in the Minkowski spacetime $\mathbb{M}^{n+1}$ no matter in the on or in the off phase. Here $t_{i}$ denotes an initial time and $t$ the current time. Indeed, Eq. (41) conveys an extremely important message that the nature of (perfect) elastoplasticity rejects time-like paths. As such the time-like metric convention has to be rejected to avoid an unreasonable negative squared length; this is the reason why we have adopted the space-like convention (22) for plasticity.

\subsection{Space-like paths versus null paths}

Regarding Eqs. (36) and (41), we may further distinguish $\dot{q}_{0}<\|\dot{\mathbf{q}}\|$ from $\dot{q}_{0}=\|\dot{\mathbf{q}}\|$ and identify the former with the space-like path and the latter with the null path, as in the following two categories:

$$
\begin{aligned}
& \dot{q}_{0}<\|\dot{\mathbf{q}}\| \Leftrightarrow \dot{\mathbf{q}}^{\mathrm{p}} \neq \dot{\mathbf{q}} \Leftrightarrow \dot{\mathbf{Q}} \neq \mathbf{0} \Leftrightarrow \text { a regular } \mathbf{Q} \text {-path } \\
& \Leftrightarrow \dot{\mathbf{X}}^{\mathrm{t}} \mathbf{g} \dot{\mathbf{X}}>0 \Leftrightarrow X^{0} \dot{\mathbf{X}} \neq \dot{X}^{0} \mathbf{X} \Leftrightarrow \mathrm{d} X>0 \Leftrightarrow \text { a space-like } \mathbf{X} \text {-path }
\end{aligned}
$$

versus

$$
\begin{aligned}
& \dot{q}_{0}=\|\dot{\mathbf{q}}\| \Leftrightarrow \dot{\mathbf{q}}^{\mathrm{p}}=\dot{\mathbf{q}} \Leftrightarrow \dot{\mathbf{Q}}=\mathbf{0} \Leftrightarrow \text { a fixed Q-point } \\
& \Leftrightarrow \dot{\mathbf{X}}^{\mathrm{t}} \mathbf{g} \dot{\mathbf{X}}=0 \Leftrightarrow X^{0} \dot{\mathbf{X}}=\dot{X}^{0} \mathbf{X} \Leftrightarrow \mathrm{d} X=0 \Leftrightarrow \text { a null } \mathbf{X} \text {-path. }
\end{aligned}
$$

A regular path refers to a path whose velocity vectors do not vanish. Most of practical situations fall into the first category of Eq. (42) - a regular path in the vector space of generalized stresses, or, correspondingly, a space-like path in the augmented stress space - in which the maximum in Eq. (36) is further sharpened to be a supremum. A few remarks on the second category of Eq. (42) are in order. In the vector space of generalized stresses the second category is a fixed point, which appears to be the projection of a null path, i.e. a ray on the null cone emanating from the origin $\mathbf{X}=\mathbf{0}$ of the augmented stress space. If $\dot{\mathbf{Q}}=\mathbf{0}$, then from Eq. (28) either $\dot{\mathbf{q}}=\mathbf{0}$, which is trivial since nothing happens, or $\dot{\mathbf{q}}=$ constant (namely, the generalized strain path is rectilinear) and

$$
\mathbf{Q}=\overline{\mathbf{Q}}:=Q^{0} \dot{\mathbf{q}} /\|\dot{\mathbf{q}}\|
$$

(namely, the generalized stress path stops and remains fixed at the point $\overline{\mathbf{Q}}$, which may be interpreted as a limit strength vector), in which $\dot{\mathbf{q}}=$ constant. The superficial impression that the generalized stress-strain curve of perfect elastoplasticity is merely an inclined straight line followed by a horizontal line in fact stems 
from the second category. All the one-dimensional cases $(n=1)$ fall into the second category, but for the dimensionality $n \geqslant 2$ the situations which fall into the second category are relatively rare [7]. The distinctive behavior for $n=1$ is rooted in topology: its yield hypersurface $\|\mathbf{Q}\|=Q^{0}$ in the vector space of generalized stresses is disconnected and contains only two points, so that in the on phase $\mathbf{Q}$ has no room to move unless to switch off.

\subsection{Relation between the Euclidean and Minkowskian lengths}

Next, we study Eq. (37), which, in view of Eqs. (9)-(11), (18), (22) and (41), amounts to

$$
\frac{\mathrm{d} X}{X^{0}}=\frac{\mathrm{d} Q}{Q^{0}},
$$

no matter in the on or in the off phase. In other words, the Minkowskian length $\mathrm{d} X$ of a differential element of a path in the augmented stress space $\mathbb{M}^{n+1}$ of $\left(X^{1}, X^{2}, \ldots, X^{n}, X^{0}\right)$ is $X^{0} / Q^{0}$ times the Euclidean length $\mathrm{d} Q$ of the corresponding differential element of the corresponding path in the generalized stress space $\mathbb{E}^{n}$ of $\left(Q^{1}, Q^{2}, \ldots, Q^{n}\right)$.

\subsection{Nguyen-Bui inequality}

Finally we study inequality (38). Constitutive models which satisfy Eq. (38) are said to be kinematically stable [9]. By Eqs. (3) and (18) we have

$$
\dot{\mathbf{q}}^{\mathrm{p}}=\frac{q_{y} \dot{X}^{0}}{\left(X^{0}\right)^{2}} \mathbf{X}^{\mathrm{s}}
$$

From Eqs. (31), (32a) and (32b) it follows that

$$
\dot{\mathbf{q}}=\frac{q_{y}}{X^{0}} \dot{\mathbf{X}}^{\mathrm{s}}
$$

Both the above two equations are valid no matter whether in the on or in the off phase. Thus Eq. (38) turns out to be

$$
\dot{X}^{0} \frac{\mathrm{d}}{\mathrm{d} t}\left[\left(\mathbf{X}^{\mathrm{s}}\right)^{\mathrm{t}} \mathbf{g}_{\mathrm{ss}} \mathbf{X}^{\mathrm{s}}\right] \geqslant 0 .
$$

In the derivations of the above three equations we have used $q_{y}>0$ and $X^{0} \geqslant 1$. Inequality (44) just says again the fact that either the "temporal" coordinate is fixed (i.e., $\mathbf{X}$ is on the closed disc for the off phase) or the "spatial" radial coordinate of the $\mathbf{X}$-path cannot be decreasing. This is rather transparent from the point of view of the geometry of the cone in the $\mathbf{X}$-space. However, its equivalent version (38) in the $\mathbf{Q}$-space is not trivial at all, its significance having been pointed out in [9].

\subsection{Paths on the cone}

From Eqs. (13), (18) and (22) it follows that

$$
\|\mathbf{Q}\|=Q^{0} \Rightarrow \mathbf{X}^{\mathrm{t}} \mathbf{g}\left(\dot{\mathbf{q}}^{\mathrm{t}}, \dot{q}_{0}\right)^{\mathrm{t}}=0 .
$$

Moreover, by Eqs. (27a), (31), (18) and (22) we can prove that

$$
\left\{\|\mathbf{Q}\|=Q^{0} \text { and } \mathbf{Q}^{\mathrm{t}} \dot{\mathbf{q}}>0\right\} \Rightarrow \mathbf{X}^{\mathrm{t}} \mathbf{g} \dot{\mathbf{X}}=0 .
$$


If the model is in the on phase (i.e., not only $\|\mathbf{Q}\|=Q^{0}$ but also $\mathbf{Q}^{t} \dot{\mathbf{q}}>0$ ), then from Eqs. (23), (45) and (46) it follows that for an $\mathbf{X}$-path on the cone, the augmented stress vector $\mathbf{X}$ is $M$-orthogonal to itself, to its tangent vector $\dot{\mathbf{X}}$ and also to its dual $\left(\dot{\mathbf{q}}^{\mathrm{t}}, \dot{q}_{0}\right)^{\mathrm{t}}$. The so-called $M$-orthogonality is an orthogonality of two $(n+1)$ dimensional vectors with respect to metric (22) in Minkowski spacetime $\mathbb{M}^{n+1}$ (see, for example [10]).

It is worth comparing Eqs. (45) and (46) with Eqs. (20), (23), (24), (40) and (42).

\subsection{Paths on the discs}

On the other hand, $X^{0}$ is frozen in the off phase as indicated by Eq. (27b) and the augmented stress $\mathbf{X}$ stays in the closed $n$-disc $\mathbb{D}^{n}$ (i.e. closed $n$-ball $\mathbb{B}^{n}$ ) on the hyperplane $X^{0}=$ constant in the space of $\left(X^{1}, X^{2}, \ldots, X^{n}, X^{0}\right)$, the hyperplane being identified to be Euclidean $n$-space $\mathbb{E}^{n}$, which is endowed with the Euclidean metric $\mathbf{I}_{n}$. In summary, the augmented stress $\mathbf{X}$ either evolves on the cone when in the on phase or stays in the discs of simultaneity, which are stacked up one by one in the interior of the cone and are glued to the cone, when in the off phase. See Fig. 2 again.

\subsection{Future pointing non-time-like paths}

The vector $\mathbf{X}(t)-\mathbf{X}\left(t_{i}\right)$ and the path $\left\{\mathbf{X}\left(t^{\prime}\right) \mid t_{i}<t^{\prime} \leqslant t\right\}$ are said to be future-pointing if $X^{0}(t)>X^{0}\left(t_{i}\right)$ strictly. Therefore, the solution to Eq. (31) with Eq. (32a) can be viewed as a future-pointing space-like or null path on the cone $\left\{\mathbf{X} \mid \mathbf{X}^{\mathrm{t}} \mathbf{g X}=0\right\}$, while the solution to Eq. (31) with Eq. (32b) is a space-like path on a closed disc of simultaneity $\left\{\mathbf{X} \mid \mathbf{X}^{\mathrm{t}} \mathbf{g X} \leqslant 0\right.$ and $\left.\dot{X}^{0}=0\right\}$. It is worth stressing that the interior of the cone is sliced into stacking discs of simultaneity tagged with different values of $X^{0}$; therefore, an admissible augmented stress can be reached either along paths in the discs of simultaneity when in the off phase or along the future-pointing space-like or null paths on the cone when in the on phase.

\section{The Lorentz group}

In this section we concentrate on the on phase to bring out internal symmetry inherent in the model in the on phase. Denoted by $I_{\text {on }}$ an open, maximal, continuous time interval during which the mechanism of plasticity is on exclusively. The solution of the augmented stress equation (31) with Eq. (32a) can be expressed in the following augmented stress transition formula:

$$
\mathbf{X}(t)=\left[\mathbf{G}(t) \mathbf{G}^{-1}\left(t_{1}\right)\right] \mathbf{X}\left(t_{1}\right), \quad \forall t, t_{1} \in I_{\mathrm{on}},
$$

in which $\mathbf{G}(t)$, known as the fundamental solution of Eq. (31), is a transformation tensor satisfying

$$
\begin{aligned}
\dot{\mathbf{G}}(t) & =\mathbf{A}(t) \mathbf{G}(t), \\
\mathbf{G}(0) & =\mathbf{I}_{n+1} .
\end{aligned}
$$

On the other hand, from Eqs. (32a) and (22) it is easy to verify that the control tensor $\mathbf{A}$ in the on phase satisfies

$$
\mathbf{A}^{\mathrm{t}} \mathbf{g}+\mathbf{g A}=\mathbf{0} .
$$

By Eqs. (50) and (48) we find

$$
\frac{\mathrm{d}}{\mathrm{d} t}\left[\mathbf{G}^{\mathrm{t}}(t) \mathbf{g} \mathbf{G}(t)\right]=\mathbf{0} .
$$


At $t=0, \mathbf{G}^{\mathrm{t}}(t) \mathbf{g G}(t)=\mathbf{I}_{n+1}^{\mathbf{t}} \mathbf{g} \mathbf{I}_{n+1}=\mathbf{g}$ from Eq. (49); thus, we prove that

$$
\mathbf{G}^{\mathrm{t}}(t) \mathbf{g} \mathbf{G}(t)=\mathbf{g}
$$

for all $t \in I_{\mathrm{on}}$. Take determinants of both sides of the above equation, getting

$$
(\operatorname{det} \mathbf{G})^{2}=1
$$

so that $\mathbf{G}$ is invertible. The 00 th component of the tensorial equation $(51)$ is $\sum_{i=1}^{n}\left(G_{0}^{i}\right)^{2}-\left(G_{0}^{0}\right)^{2}=-1$, from which

$$
\left(G_{0}^{0}\right)^{2} \geqslant 1
$$

Here $G_{j}^{i}, i, j=1,2, \ldots, n, 0$, is the $i j$ th mixed component of the tensor $\mathbf{G}$. Since $\operatorname{det} \mathbf{G}=-1$ or $G_{0}^{0} \leqslant-1$ would violate Eq. (49), it turns out that

$$
\begin{aligned}
& \operatorname{det} \mathbf{G}=1, \\
& G_{0}^{0} \geqslant 1 .
\end{aligned}
$$

In summary, $\mathbf{G}$ has the three characteristic properties explicitly expressed by Eqs. (51), (54) and (55).

Recall that the complete homogeneous Lorentz group $\mathrm{O}(n, 1)$ is the group of all invertible linear transformations in Minkowski spacetime which leave the Minkowski metric invariant, and that the proper orthochronous Lorentz group $\mathrm{SO}_{0}(n, 1)$ is a subgroup of $\mathrm{O}(n, 1)$ in which the transformations are proper (i.e., orientation preserving, namely the determinants of the transformations being +1 ) and orthochronous (i.e., time-orientation preserving, namely the 00th entry of the matrix representations of the transformations being positive); see, for example, [11]. Hence, in view of the three characteristic properties we conclude that the fundamental solution $\mathbf{G}$ belongs to the proper orthochronous Lorentz group $\mathrm{SO}_{0}(n, 1)$. Therefore, the tensor-valued function $\mathbf{G}(t)$ of time $t \in I_{\text {on }}$ may be viewed as a connected path of the Lorentz group. Furthermore, by Eq. (50), $\mathbf{A}$ is an element of the real Lie algebra so( $n, 1)$ of the Lorentz group $\operatorname{SO}_{0}(n, 1)$. Thereby the algebraic and topological properties of the proper orthochronous Lorentz group are shared by the perfect elastoplasticity model (1)-(6).

From Eq. (27a), $\dot{X}^{0}>0$ strictly when the mechanism of plasticity is on; hence, ${ }^{6}$

$$
X^{0}(t)>X^{0}\left(t_{1}\right)>X^{0}\left(t_{0}\right)=1, \quad \forall t>t_{1}>t_{0}, t, t_{1} \in I_{\text {on }}
$$

which means that in the sense of irreversibility there exists future-pointing time-orientation from the augmented stress $\mathbf{X}\left(t_{1}\right)$ to $\mathbf{X}(t)$. Moreover, such time-orientation is a causal one, because the augmented stress transition Eq. (47) and inequality (56) establish a causality relation between the two augmented stress vectors $\mathbf{X}\left(t_{1}\right)$ and $\mathbf{X}(t)$ in the sense that the preceding augmented stress vector $\mathbf{X}\left(t_{1}\right)$ influences the following augmented stress vector $\mathbf{X}(t)$ according to Eq. (47). Accordingly, the augmented stress vector $\mathbf{X}\left(t_{1}\right)$ chronologically and causally precedes the augmented stress vector $\mathbf{X}(t)$. This is indeed a common property for all models with inherent symmetry of the proper orthochronous Lorentz group. By this symmetry a core connection among irreversibility, the time arrow of evolution, and causality has thus been established for plasticity in the on phase.

\footnotetext{
${ }^{6}$ From Eqs. (5), (10) and (18) it follows that $X^{0}(t) \geqslant X^{0}\left(t^{\prime}\right) \geqslant X^{0}\left(t_{i}\right) \geqslant X^{0}\left(t_{0}\right)=1$ for all $t \geqslant t^{\prime} \geqslant t_{i} \geqslant t_{0}$, applicable to both the on and off phases. Recall that $t_{0}$ is the zero-value time at which all relevant values including $q_{0}\left(t_{0}\right)=0$.
} 


\section{Projective realization}

Relation (47) of the group of transformations can be made to be more useful especially when interpreted in terms of generalized stresses. To elaborate, we solve Eq. (51) for the inverse

$$
\mathbf{G}^{-1}=\mathbf{g G}^{\mathbf{t}} \mathbf{g}
$$

and partition $\mathbf{G}$ as

$$
\mathbf{G}=\left[\begin{array}{ll}
\mathbf{G}_{\mathrm{s}}^{\mathrm{s}} & \mathbf{G}_{0}^{\mathrm{s}} \\
\mathbf{G}_{\mathrm{s}}^{0} & G_{0}^{0}
\end{array}\right]
$$

Thus, Eq. (47) is partitioned into

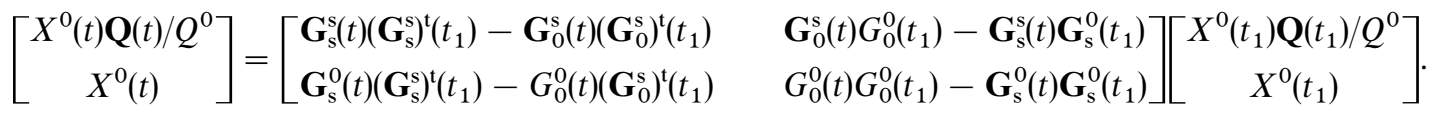

Dividing the first row by the second row we have

$$
\begin{aligned}
& \mathbf{Q}(t)=\left\{\left[\mathbf{G}_{\mathbf{s}}^{\mathbf{s}}(t)\left(\mathbf{G}_{\mathbf{s}}^{\mathrm{s}}\right)^{\mathrm{t}}\left(t_{1}\right)-\mathbf{G}_{0}^{\mathrm{s}}(t)\left(\mathbf{G}_{0}^{\mathbf{s}}\right)^{\mathrm{t}}\left(t_{1}\right)\right] \mathbf{Q}\left(t_{1}\right) Q^{0}+\left[\mathbf{G}_{0}^{\mathrm{s}}(t) G_{0}^{0}\left(t_{1}\right)-\mathbf{G}_{\mathbf{s}}^{\mathbf{s}}(t) \mathbf{G}_{\mathbf{s}}^{0}\left(t_{1}\right)\right]\left(Q^{0}\right)^{2}\right\} / \\
&\left\{\left[\mathbf{G}_{\mathbf{s}}^{0}(t)\left(\mathbf{G}_{\mathbf{s}}^{\mathbf{s}}\right)^{\mathbf{t}}\left(t_{1}\right)-G_{0}^{0}(t)\left(\mathbf{G}_{0}^{\mathbf{s}}\right)^{\mathrm{t}}\left(t_{1}\right)\right] \mathbf{Q}\left(t_{1}\right)+\left[G_{0}^{0}(t) G_{0}^{0}\left(t_{1}\right)-\mathbf{G}_{\mathbf{s}}^{0}(t) \mathbf{G}_{\mathbf{s}}^{0}\left(t_{1}\right)\right] Q^{0}\right\} .
\end{aligned}
$$

The transformation is known as a projective unimodular transformation, which maps $\mathbf{Q}\left(t_{1}\right)$ to the current response $\mathbf{Q}(t)$. In fact the generalized stress $\mathbf{Q}$, a vector with $n$ components supposed to be observable, is a projective realization of the vector $\mathbf{X}$ which has $n+1$ homogeneous coordinates.

Contrary to Eq. (60) in the on phase, the mapping in the off phase is very simple,

$$
\mathbf{Q}(t)=\mathbf{Q}\left(t_{1}\right)+k_{\mathrm{e}}\left[\mathbf{q}(t)-\mathbf{q}\left(t_{1}\right)\right],
$$

where $\mathbf{Q}\left(t_{1}\right)$ is the starting generalized stress vector at time $t_{1}$ in the off phase. It may be recast to the transformation of augmented stress vectors:

$$
\left[\begin{array}{c}
X^{0}(t) \mathbf{Q}(t) / Q^{0} \\
X^{0}(t)
\end{array}\right]=\mathbf{G}(t) \mathbf{G}^{-1}\left(t_{1}\right)\left[\begin{array}{c}
X^{0}\left(t_{1}\right) \mathbf{Q}\left(t_{1}\right) / Q^{0} \\
X^{0}\left(t_{1}\right)
\end{array}\right]
$$

but with

$$
\mathbf{G}(t)=\left[\begin{array}{cc}
\mathbf{I}_{n} & \mathbf{q}(t) / q_{\mathrm{y}} \\
\mathbf{0}^{\mathrm{t}} & 1
\end{array}\right] .
$$

Even such an off-phase transformation of augmented stresses exists and is invertible; it is no longer an element of the Lorentz group because such $\mathbf{G}$ does not satisfy Eq. (51) although $\operatorname{det} \mathbf{G}=1$ remains to hold and $G_{0}^{0}=1$. It belongs to a translation group $T(n)$ on $\mathbb{B}^{n}$ of $\mathbb{E}^{n}$. The change from a transformation of the Lorentz group in the on phase to a non-Lorentzian transformation in the off phase indicates that internal symmetry switches from one kind to another.

\section{Exact solutions for rectilinear paths}

Consider a rectilinear generalized strain path

$$
\mathbf{q}(t)=\mathbf{q}\left(t_{1}\right)+\left(t-t_{1}\right) \dot{\mathbf{q}}
$$


with non-zero constant rate

$$
\dot{\mathbf{q}}=\text { constant } \neq \mathbf{0},
$$

starting from $\mathbf{q}\left(t_{1}\right)$ at time $t_{1}$. The constitutive response can be determined exactly [7], and it may be recast in the form of Eq. (47) with the augmented stress transition tensor for the on phase being

$$
\mathbf{G}(t) \mathbf{G}^{-1}\left(t_{1}\right)=\left[\begin{array}{cc}
\mathbf{I}_{n}+\left((a-1) /\|\dot{\mathbf{q}}\|^{2}\right) \dot{\mathbf{q}} \dot{\mathbf{q}}^{\mathrm{t}} & b \dot{\mathbf{q}} /\|\dot{\mathbf{q}}\| \\
b \dot{\mathbf{q}}^{\mathrm{t}} /\|\dot{\mathbf{q}}\| & a
\end{array}\right],
$$

in which

$$
a:=\cosh \left[\left(t-t_{1}\right)\|\dot{\mathbf{q}}\| / q_{\mathrm{y}}\right], \quad b:=\sinh \left[\left(t-t_{1}\right)\|\dot{\mathbf{q}}\| / q_{\mathrm{y}}\right] .
$$

To examine the group properties, we find the fundamental solution as

$$
\mathbf{G}(t)=\left[\begin{array}{cc}
\mathbf{I}_{n}+\left((a-1) /\|\dot{\mathbf{q}}\|^{2}\right) \dot{\mathbf{q}} \dot{\mathbf{q}}^{\mathrm{t}} & b \dot{\mathbf{q}} /\|\dot{\mathbf{q}}\| \\
b \dot{\mathbf{q}}^{\mathrm{t}} /\|\dot{\mathbf{q}}\| & a
\end{array}\right],
$$

but with

$$
a:=\cosh \left(t\|\dot{\mathbf{q}}\| / q_{\mathrm{y}}\right), \quad b:=\sinh \left(t\|\dot{\mathbf{q}}\| / q_{\mathrm{y}}\right) .
$$

It is not difficult to see that the above $\mathbf{G}$ satisfies Eqs. (51), (54) and (55); therefore, the curve $\mathbf{G}(t)$ going through the identity $\mathbf{G}(0)=\mathbf{I}$ at $t=0$ is a one-parameter subgroup of the Lorentz group $\mathrm{SO}_{0}(n, 1)$. The projective realization developed in Section 8 becomes for this case

$$
\mathbf{Q}(t)=\frac{Q^{0}\left[\mathbf{Q}\left(t_{1}\right)+\left((a-1) /\|\dot{\mathbf{q}}\|^{2}\right) \dot{\mathbf{q}}^{\mathrm{t}} \mathbf{Q}\left(t_{1}\right) \dot{\mathbf{q}}\right]+b\left(Q^{0}\right)^{2} \dot{\mathbf{q}} /\|\dot{\mathbf{q}}\|}{b \dot{\mathbf{q}}^{\mathrm{t}} \mathbf{Q}\left(t_{1}\right) /\|\dot{\mathbf{q}}\|+a Q^{0}} .
$$

This is the generalized stress response for the on phase, while in the off phase it is replaced by Eq. (61).

\section{Group preserving schemes for general paths}

Now let us consider general paths of generalized strain inputs and find their responses. To devise numerical schemes for time marching, let us denote the time increment by $\Delta t$ and develop maps to update $\mathbf{Q}(t)$ to the next time step $\mathbf{Q}(t+\Delta t)$.

\subsection{A scheme for piecewise rectilinear paths}

We may approximate a general path by a piecewise rectilinear path. Referring to Eq. (64), we obtain the desired map

$$
\mathbf{G}(t+\Delta t) \mathbf{G}^{-1}(t)=\left[\begin{array}{cc}
\mathbf{I}_{n}+\left((a-1) /\|\dot{\mathbf{q}}\|^{2}\right) \dot{\mathbf{q}} \dot{\mathbf{q}}^{\mathrm{t}} & b \dot{\mathbf{q}} /\|\dot{\mathbf{q}}\| \\
b \dot{\mathbf{q}}^{\mathrm{t}} /\|\dot{\mathbf{q}}\| & a
\end{array}\right]
$$

with

$$
a:=\cosh \left(\Delta t\|\dot{\mathbf{q}}\| / q_{\mathrm{y}}\right), \quad b:=\sinh \left(\Delta t\|\dot{\mathbf{q}}\| / q_{\mathrm{y}}\right) .
$$

Thus a numerical scheme for the on phase may be devised as follows: for each time increment first calculate the map (69), then update the augmented stress vector by

$$
\mathbf{X}(t+\Delta t)=\mathbf{G}(t+\Delta t) \mathbf{G}^{-1}(t) \mathbf{X}(t)
$$

and finally calculate the generalized stress vector $\mathbf{Q}(t+\Delta t)$ via Eq. (18). 


\subsection{Cayley transform scheme for general paths}

For $\mathbf{A} \in \operatorname{so}(n, 1)$ the group generated is known as a Lorentz rotation or boost group. For the Lorentz rotation, the Cayley transform

$$
\operatorname{Cay}(\kappa \mathbf{A})=(\mathbf{I}-\kappa \mathbf{A})^{-1}(\mathbf{I}+\kappa \mathbf{A})
$$

is also a map from $\mathbf{A}$ to an element of $\operatorname{SO}_{0}(n, 1)$ for $\kappa \in \mathbb{R}$ and $\kappa^{2}<q_{\mathbf{y}}^{2} /\|\dot{\mathbf{q}}\|^{2}$. Substituting Eq. (32a) for $\mathbf{A}$ in the above equation yields

$$
\operatorname{Cay}(\kappa \mathbf{A})=\left[\begin{array}{cc}
\mathbf{I}_{n}+2 \kappa^{2} /\left(q_{\mathrm{y}}^{2}-\kappa^{2}\|\dot{\mathbf{q}}\|^{2}\right) \dot{\mathbf{q}} \dot{\mathbf{q}}^{\mathrm{t}} & 2 \kappa q_{\mathrm{y}} /\left(q_{\mathrm{y}}^{2}-\kappa^{2}\|\dot{\mathbf{q}}\|^{2}\right) \dot{\mathbf{q}} \\
2 \kappa q_{\mathrm{y}} /\left(q_{\mathrm{y}}^{2}-\kappa^{2}\|\dot{\mathbf{q}}\|^{2}\right) \dot{\mathbf{q}}^{\mathrm{t}} & \left(q_{\mathrm{y}}^{2}+\kappa^{2}\|\dot{\mathbf{q}}\|^{2}\right) /\left(q_{\mathrm{y}}^{2}-\kappa^{2}\|\dot{\mathbf{q}}\|^{2}\right)
\end{array}\right] .
$$

Thus we devise the Cayley transform scheme as follows: for each time increment first calculate the Cayley transform (72), then update the augmented stress vector by

$$
\mathbf{X}(t+\Delta t)=\operatorname{Cay}(\kappa \mathbf{A}(t)) \mathbf{X}(t),
$$

and finally calculate the generalized stress vector $\mathbf{Q}(t+\Delta t)$ via Eq. (18).

\subsection{Comments on the two schemes}

Comment 1. For the above two schemes, we must calculate $\mathbf{X}$ at each time step but need not calculate $\mathbf{Q}$ at every time step unless it is needed. We thus accelerate time marching and more efficiently obtain the generalized stress response $\mathbf{Q}$ only at the target time instant.

Comment 2. Note that maps (69) and (72) satisfy the three characteristic properties (51), (54) and (55) of $\mathrm{SO}_{0}(n, 1)$; hence, the resultant schemes are both group preserving schemes. For the two schemes, the Lorentz group property ensures that, at every time step in the on phase, the maps update the augmented stress point to be always on the cone. As projective realizations, the generalized stress points in the on phase are, therefore, always located on the yield hypersphere $\mathbb{S}^{n-1}$, that is,

$$
\|\mathbf{Q}\|=Q^{0} \text { in the on phase. }
$$

This symmetry group property is essential for computational plasticity to completely avoid iterations, predictions and corrections, etc., in order to fulfill the consistency condition.

Comment 3. A remarkable fact specific to a Lorentz rotation is that an exponential map of $\mathbf{A}$ admits a closed-form representation given by the following formula:

$$
\exp (\Delta t \mathbf{A})=\left[\begin{array}{cc}
\mathbf{I}_{n}+\left((a-1) /\|\dot{\mathbf{q}}\|^{2}\right) \dot{\mathbf{q}} \dot{\mathbf{q}}^{\mathrm{t}} & b \dot{\mathbf{q}} /\|\dot{\mathbf{q}}\| \\
b \dot{\mathbf{q}}^{\mathrm{t}} /\|\dot{\mathbf{q}}\| & a
\end{array}\right]
$$

with $a$ and $b$ given by Eq. (70). Notice that exponential map (73) is just the $\mathbf{G}(t+\Delta t) \mathbf{G}^{-1}(t)$ in Eq. (69). In terms of $\mathbf{A}$ given in Eq. (32a), the exponential map (73) for $\mathrm{SO}_{0}(n, 1)$ acting on $\mathbb{M}^{n+1}$ becomes

$$
\exp (\Delta t \mathbf{A})=\mathbf{I}_{n+1}+\sinh \left(\frac{\|\dot{\mathbf{q}}\|}{q_{\mathrm{y}}} \Delta t\right) \frac{q_{\mathrm{y}} \mathbf{A}}{\|\dot{\mathbf{q}}\|}+\left[\cosh \left(\frac{\|\dot{\mathbf{q}}\|}{q_{\mathrm{y}}} \Delta t\right)-1\right] \frac{q_{\mathrm{y}}^{2} \mathbf{A}^{2}}{\|\dot{\mathbf{q}}\|^{2}} .
$$

This is similar to the classical formula of Euler-Rodrigues for $\mathrm{SO}(3)$ acting on $\mathbb{E}^{3}$,

$$
\exp (\Delta t \mathbf{W})=\mathbf{I}_{3}+\sin (w \Delta t) \frac{\mathbf{W}}{w}+[1-\cos (w \Delta t)] \frac{\mathbf{W}^{2}}{w^{2}},
$$


where $\mathbf{W}$ is a three-dimensional spin tensor and $w$ is the Euclidean norm of the axial vector of $\mathbf{W}$.

Comment 4. If one takes

$$
\kappa=\frac{q_{\mathrm{y}}\left[1-\cosh \left(\Delta t\|\dot{\mathbf{q}}\| / q_{\mathrm{y}}\right)\right]}{\|\dot{\mathbf{q}}\| \sinh \left(\Delta t\|\dot{\mathbf{q}}\| / q_{\mathrm{y}}\right)},
$$

the Cayley transform (72) is exactly equal to exponential map (73), that is,

$$
\operatorname{Cay}(\kappa \mathbf{A})=\exp (\Delta t \mathbf{A}) \text {. }
$$

If one takes

$$
\kappa=\frac{\Delta t}{2}
$$

the resulting is a time-centered Euler scheme. Therefore, the Cayley transform scheme accommodates more general input paths than piecewise rectilinear paths.

Comment 5. Similar to the above statement "Eq. (76) if Eq. (75)" for $\operatorname{SO}_{0}(n, 1)$ acting on $\mathbb{M}^{n+1}$, a statement for $\mathrm{SO}(3)$ acting on $\mathbb{E}^{3}$ has been derived in [12] as follows:

$$
\operatorname{Cay}(\kappa \mathbf{W})=\exp (\Delta t \mathbf{W}) \quad \text { if } \quad \kappa=\frac{2 \tan (w \Delta t / 2)}{w \Delta t} .
$$

\subsection{Example}

Fig. 3 displays an example of comparison between the exact solutions in Section 9 and the results calculated using the group preserving scheme in Section 10.1 for the model subjected to an input of a cyclic triangular path in two dimensions as shown in Fig. 3a. The first cycle consists of three pieces 1, 2, 3, the second cycle consisting of pieces 4, 5, 6 repeats in the generalized strain space the locus of the first cycle of pieces 1, 2 and 3, and so forth. The material constants used are $k_{\mathrm{e}}=50000 \mathrm{MPa}$ and $Q^{0}=400 \mathrm{MPa}$. Only the responses of the first two cycles are displayed because after those the responses were found to be almost repeated and stabilized. The results shown include the generalized stress path in Fig. 3b, hysteresis loops in Figs. 3e and f, time histories of generalized stresses in Fig. 3c. The response graph of the generalized stress path in Fig. $3 \mathrm{~b}$ as can be seen is very different from the input graph of the generalized strain path in Fig. 3a. One of two main features is that the generalized strain path is closed, but the corresponding generalized stress response has an open path. The points marked by (1), (4) and (7) in Fig. 3a are the same generalized strain points; however, the resulting generalized stress path 123456 as shown in Fig. 3b starts at, passes through and ends at three different generalized stress points marked by (1), (4) and (7), respectively. The other feature is that the generalized strain path is composed of straight lines, but the corresponding generalized stress response has straight-line paths in the off phase but circular paths in the on phase. It is obvious that the group-preserving scheme gave very accurate responses and supplied a completely faithful result of the consistency condition.

\section{Remarks on some generalizations}

An element $\mathbf{A}$ of the real Lie algebra so(n,1) which satisfies Eq. (50) has the general form

$$
\mathbf{A}=\left[\begin{array}{ll}
\mathbf{A}_{\mathrm{s}}^{\mathrm{s}} & \mathbf{A}_{0}^{\mathrm{s}} \\
\mathbf{A}_{\mathrm{s}}^{0} & 0
\end{array}\right]
$$




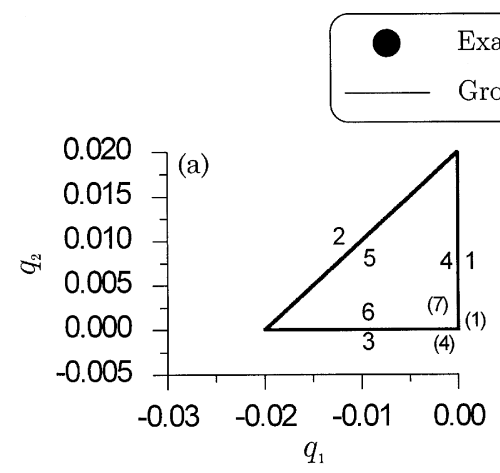

(c)
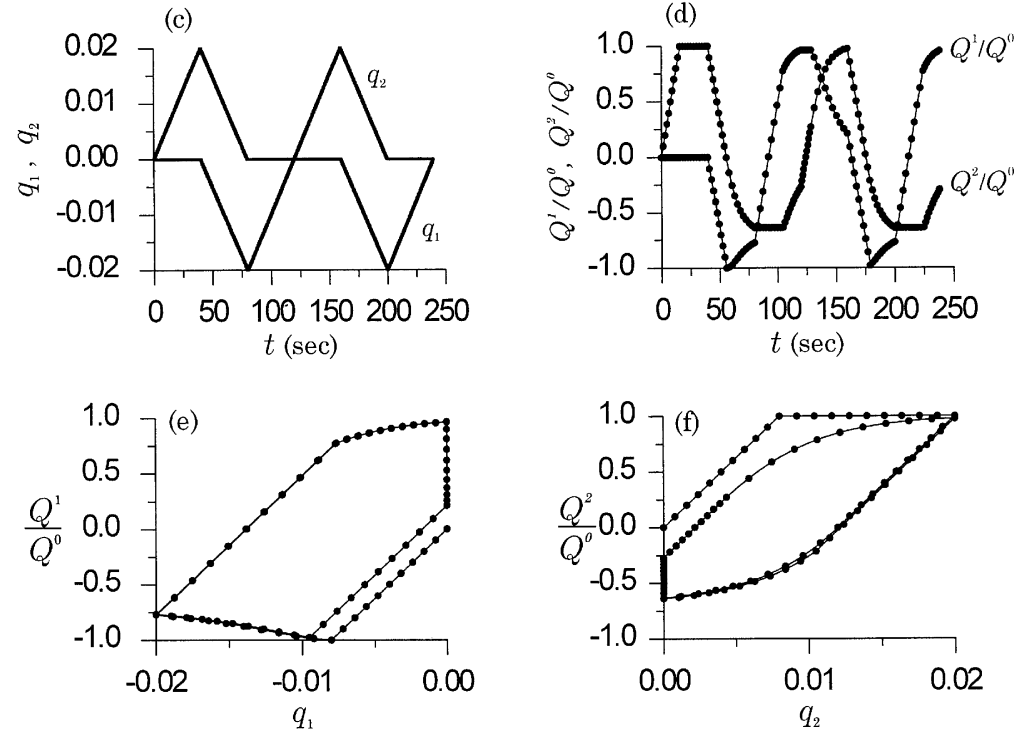

Fig. 3. Comparison between the exact solutions and the results calculated using the group preserving scheme for the perfect elastoplastic model in two dimensions under a cyclic triangular generalized strain path: (a) input of the generalized strain path; (b) output of the generalized stress path; time histories of (c) generalized strains and (d) generalized stresses; hysteresis loops of (e) $Q^{1}$ versus $q_{1}$ and (f) $Q^{2}$ versus $q_{2}$.

with the properties

$$
\left(\mathbf{A}_{\mathrm{s}}^{\mathrm{s}}\right)^{\mathrm{t}}=-\mathbf{A}_{\mathrm{s}}^{\mathrm{s}}, \quad \mathbf{A}_{\mathrm{s}}^{0}=\left(\mathbf{A}_{0}^{\mathrm{s}}\right)^{\mathrm{t}}
$$

But the earlier form of $\mathbf{A}$ as given in Eqs. (32a) and (32b) has zero $\mathbf{A}_{\mathrm{s}}^{\mathrm{s}}$ and is, therefore, less general than it may. We may generalize our model by including a non-vanishing, skew-symmetric tensor $\mathbf{A}_{\mathrm{s}}^{\mathrm{s}}$ if we no longer assume negligibly small spinning but instead consider large deformation and rotation [13].

A straightforward generalization of the Lorentz group $\mathrm{SO}_{0}(n, 1)$ is a Poincare group, which is a semi-direct product of a translation group with the Lorentz group. In this way we can take the (linear [14] or non-linear) kinematic hardening-softening effect into consideration. In the on phase the augmented stress vectors remain on the cone. A generalization for this is to replace the cone by a hyperboloid, resulting in a model capable of accounting for the isotropic hardening-softening. A simultaneous generalization of the two thus renders mixed hardening. 
Since the three generalizations briefly mentioned above do not alter the core of the linear representation in the augmented stress space, the group preserving schemes for general paths presented in Section 10 and the exact solutions for rectilinear paths presented in Section 9 will be still applicable, after minor modifications and extensions, to the resulting more sophisticated models; in particular, the generalized stress point at every time step will be located automatically on the yield surface even when the yield surface moves kinematically and meanwhile expands (or contracts) isotropically. Note that the linearity in the augmented stress space and the automatic fulfillment of the consistency condition are essential for computational plasticity and both remain valid even after the suggested generalizations.

\section{Conclusions}

In this paper we have converted the constitutive model of perfect elastoplasticity to a two-phase system of linear differential equations in the $(n+1)$-dimensional augmented stress space of $\mathbf{X}$. In the augmented stress space not only the non-linearity of the model was unfolded, but also an intrinsic spacetime structure of the Minkowskian type was brought out. The control tensor A which contains the generalized strain rate vector $\dot{\mathbf{q}}$ was shown to be an element of the real Lie algebra so $(n, 1)$ of the proper orthochronous Lorentz group $\mathrm{SO}_{0}(n, 1)$, and the fundamental solution $\mathbf{G}$ of the linear differential system (31) was proved to be an element of the Lorentz group, so that the causality relation of the augmented stresses was verified. Based on the Lorentz group transformation (between $\mathbf{X}(t)$ and $\mathbf{X}\left(t_{1}\right)$ ) in the augmented stress space a projective transformation (between $\mathbf{Q}(t)$ and $\mathbf{Q}\left(t_{1}\right)$ ) in the generalized stress space was identified such that the evolution rule of the generalized stress vectors became available. To account for both the on and off phases a composite space endowed with a Minkowskian metric on the cone but with a Euclidean metric on each of the discs inside the cone may be constructed. As a result the perfect elastoplasticity model possesses two kinds of symmetry - $T(n)$ (the translation group) in the off phase and $\operatorname{PSO}_{0}(n, 1)$ (the projective realization of the proper orthochronous Lorentz group) in the on phase and has symmetry switching between the two depending on the generalized strain path input.

Using the symmetry group properties we have developed a Cayley transformation and devised the Cayley transform scheme, which possesses the crucial property that the generalized stress points are updated automatically on the yield surface at every time step. The scheme is applicable to general paths, while for rectilinear paths we have obtained exact solutions (68) and (61). These results (and their generalizations to the suggested more sophisticated models) are due to the symmetry group properties, among which the linearity in the augmented stress space and the automatic fulfillment of the consistency condition are particularly valuable for computational plasticity. An understanding of the internal symmetries in the underlying structure is not only important in its own right but also beneficial to computation.

\section{Acknowledgements}

The financial support provided by the National Science Council under the Grant NSC 85-2211-E-002-001 is gratefully acknowledged. A preliminary version of the work presented here appeared as Chapter 2, pp. 7-29, of the report [15].

\section{References}

[1] W. Prager, The theory of plasticity: A survey of recent achievements, Proc. Inst. Mech. Engng 169 (1955) 41-57.

[2] W. Prager, A new method of analyzing stresses and strains in work-hardening plastic solids, J. Appl. Mech. 23 (1956) 493-496. 
[3] L. Prandtl, Spannungsverteilung in plastischen kœrpern. In Proceedings of the 1st International Congress on Applied Mechanics, Delft, 1924, pp. 43-54.

[4] E. Reuss, Beruecksichtigung der elastischen formaenderungen in der plastizitaetstheorie, Zeits. angew. Math. Mech. (ZAMM) 10 (1930) 266-274.

[5] H.-K. Hong, C.-S. Liu, Prandtl-Reuss elastoplasticity: On-off switch and superposition formulae, Int. J. Solids Struct. 34 (1997) 4281-4304.

[6] I.M. Gel'fand, M.I. Graev, N.Y. Vilenkin, Generalized Functions, Integral Geometry and Representation Theory, Vol. 5, Academic Press, New York, 1966.

[7] H.-K. Hong, C.-S. Liu, On behavior of perfect elastoplasticity under rectilinear paths, Int. J. Solids Struct. 35 (1998) $3539-3571$.

[8] H.-K. Hong, J.-K. Liou, Integral-equation representations of flow elastoplasticity derived from rate-equation models, Acta Mech. 96 (1993) 181-202.

[9] Q.S. Nguyen, H.D. Bui, Sur les matériaux à écrouissage positif ou négatif, J. de Mécanique 13 (1974) $321-342$.

[10] G.L. Naber, The Geometry of Minkowski Spacetime, Springer, New York, 1992.

[11] J.F. Cornwell, Group Theory in Physics, Vol. 2, Academic Press, London, 1984.

[12] D. Lewis, J.C. Simo, Conserving algorithms for the dynamics of Hamiltonian systems on Lie groups, J. Non-linear Sci. 4 (1994) 253-299.

[13] H.-K. Hong, C.-S. Liu, Lorentz group $\mathrm{SO}_{0}(5,1)$ for perfect elastoplasticity with large deformation and a consistency numerical scheme, Int. J. Non-Linear Mech. 34 (1999) 1113-1130.

[14] H.-K. Hong, C.-S. Liu, Internal symmetry in bilinear elastoplasticity, Int. J. Non-Linear Mech. 34 (1999) $279-288$.

[15] H.-K. Hong, C.-S. Liu, Analyses, experimentation and identification of dynamic system of elastoplasticity (2), Report prepared for the National Science Council Project No. NSC85-2211-E-002-001, Department of Civil Engineering, Taiwan University, Taipei, 1996. 